\title{
Using the FORTH language in real-time computer applications
}

\author{
DOUGLAS LEA \\ University of New Hampshire, Durham, New Hampshire 03824
}

\begin{abstract}
The FORTH computer language is described, with emphasis on aspects relevant to its use in the real-time control of psychological experiments via microcomputers. Among FORTH's advantages are speed, compactness, and flexibility. Potential disadvantages include unusual syntax and lack of file-oriented disk access. Two sample FORTH-based systems are described.
\end{abstract}

FORTH is a microcomputer language that combines, within a simple, compact framework, the advantages of different languages such as BASIC, PASCAL, LISP, and pure assembly language. Several general accounts of FORTH have appeared recently (e.g., Katzan, 1981; Ouverson, 1981; Williams, 1980). Readers should refer to such works for comprehensive introductions. Here, I will discuss some aspects of FORTH that make it attractive for real-time systems.

(1) FORTH is fast. It is a truly compiled language, although it is unusual in its method of compilation. FORTH compiles code by threading together previously compiled routines (called definitions, or words) and executes them by jumping from routine to routine. In a sense, all FORTH words are subroutines. The execution speed of FORTH programs is typically faster than that of similar programs in PASCAL or most other languages available on microcomputers. FORTH is slower than raw assembly or code generated from some languages using very efficient compilers because of FORTH's frequent jumps between routines, although each jump requires only two machine instructions on most versions.

(2) FORTH is compact. Threaded code occupies very little space, since only the address, not the actual instructions, of each routine is ever compiled within new definitions. This means that the codes for common primitive operations such as " + " and "/" exist only once in any FORTH system. Also, the compilation routines required to thread code are short and simple. An entire FORTH system, with user programs, can reside in less than $8 \mathrm{~KB}$ of memory, although $16 \mathrm{~KB}$ is a practical lower limit for any FORTH system not dedicated to a single simple task.

(3) FORTH is an interpretive language. Instructions,

Reprints may be obtained from Douglas Lea, Department of Psychology, University of New Hampshire, Durham, New Hampshire 03824. The MINC system described here is supported in part by NSF Grant BNS 76-11028 to J. A. Nevin. The TRS-80 system is supported in part by NIMH Grant 1-R03-MH-3459701 to $\mathrm{C}$. Mandell. I thank all connected with these laboratories. once defined, are executed interpretively. FORTH has two modes: execution and compilation. The difference between these is almost identical to the difference between typing unnumbered BASIC instructions with immediate results and writing a series of numbered BASIC statements that serve as a program.

(4) FORTH allows pure assembly code routines. Most versions contain their own assemblers, allowing programmers to write machine code routines to perform time-critical and/or machine-dependent tasks (e.g., interrupt handling) without leaving the FORTH language. After compilation, assembly code routines are treated like any other FORTH words.

(5) FORTH is a structured language. Like PASCAL, $\mathrm{C}$, and similar languages, FORTH includes DO, WHILE, and UNTIL loops, IF ...ELSE and CASE structures, and recursion, but not GOTO statements or any other unstructured branching devices.

(6) FORTH has an unusual syntax. It operates entirely in reverse Polish (or postfix) notation. All FORTH words operate upon a standard first-in/last-out stack, much like an RPN calculator. However, while the use of RPN for arithmetic expressions may be familiar to most programmers, FORTH's reliance on postfix for all of its operations usually requires some adjustment. For example,

$$
\mathrm{X} \mathrm{Y} 0=\operatorname{IF} 2+\operatorname{ELSE} 4 * \text { ENDIF }
$$

compares the constant $Y$ with zero, and if the comparison results in a Boolean TRUE, it adds two to the constant $\mathrm{X}$ or, otherwise, multiplies $\mathrm{X}$ by four, leaving the result of the computation on the stack in either case. The syntactic difference between FORTH and infix notation languages like PASCAL is basically the same as the difference between such languages and prefix notation languages like LISP. In fact, because of the similarities between postfix and prefix, LISP programmers should find FORTH a very easy language to learn.

(7) FORTH is flexible. New program structures and data types (such as virtual arrays, strings, linked lists, 
bit-wise accessible memory space, CASE constructions with built-in table look-ups, etc.) may be defined and then used like built-in "defining words." A defining word is like a declaration statement in other languages. For example, the defining word VARIABLE is used to create new named variables. Because of this, and the fact that FORTH allows the user total access to and control of his machine (not one "primitive" operation is hidden from the user), most FORTH systems soon become personalized to the needs and tastes of their programmers.

(8) FORTH is powerful. Once adapted to FORTH's postfix conventions, the advantages of RPN over infix in arithmetic computations extend to most other operations as well. For example, many routines can perform their tasks simply by manipulating numbers on the stack. This all but eliminates the need for temporary variables. Also, FORTH encourages users to build more and more powerful definitions by combining other definitions, leading to single-word utility routines that perform complex tasks. While most other languages allow such construction of subroutine libraries, FORTH's structure makes this practice the most natural method of programming. In fact, other programming strategies often lead the user to face a minor limitation of FORTH: FORTH does not allow forward references. New definitions may invoke only words that have previously been defined. This means that a main program can be entered only after all of its constituent routines have been compiled successfully. This forces programmers to write and debug programs from the bottom up, which, in conjunction with the emphasis on top-down design implicit in any structured language, typically shortens the time between thinking up and running programs. In cases of absolute necessity, knowledgeable programmers can "patch in" forward references.

(9) FORTH is not a file-oriented language. FORTH treats all disk space as virtual memory, accessible in 1-KB "blocks" by reading the needed disk contents into buffers. This lack of file-oriented disk $\mathrm{I} / \mathrm{O}$ is probably the single biggest obstruction for users who would like to switch immediately from another language to FORTH. Since FORTH cannot create data files, and so on, for use in existing programs, all such programs have to be rewritten in FORTH. There are a few ways around this. For example, on a TRS-80, we have created dummy files in BASIC and then programmed FORTH to read to or write from the allotted disk space. However, most programmers have found that, on small systems, FORTH's "manual" file management procedures are usually more sensible than the use of automated disk operating systems. Moreover, it is not difficult to write customized disk operating routines that incorporate the useful features of any file management system. (At least one $\mathrm{CP} / \mathrm{M}$-compatible file-oriented version of FORTH, with which I have no experience, has appeared on the market.)

\section{SAMPLE FORTH SYSTEMS}

In one laboratory, we use a DEC LSI-11/2 MINC with $60 \mathrm{~KB}$ of memory and peripheral laboratory I/O modules. Operation is under a version of the FORTH Interest Group's PDP-11 FIG-FORTH 1.0, with extensive modifications that take advantage of MINC-specific characteristics, as well as other minor coding improvements and alterations.

We currently run six simultaneous animal experiments involving discrimination, memory, and reinforcement schedule performance. These are strung together as a single background job and driven by a real-time clock-based interrupt every $25 \mathrm{msec}$. Processing time averages less than $5 \mathrm{msec} /$ interrupt. MINC users operating under BASIC or FORTRAN may be surprised to learn that this core-resident background job leaves about $25 \mathrm{~KB}$ of free program space after loading. This space is used by foreground jobs, for which we presently have programs for text processing (using a VT100 screen-based file editor/text formatter), graphics plotting, "smart-terminal" communication with a main-frame computer, and data analysis (including a library of floating-point mathematical routines), along with over 100 pages of on-line documentation files.

Writing timeshared real-time experimental control programs on this system is not much different from that on systems employing any other structured language. Each experiment is broken down into separate routines that perform the tasks necessary between "waiting" states (e.g., waiting for a response to occur or a time delay to elapse) and then exit. The experiment is then driven by a main program consisting mainly of a CASE statement, directing execution to the proper routine during each interrupt.

Each experiment control program is written entirely in FORTH. Only a few general utility routines (e.g., those used to count down and signal time delays) have been coded in assembly. This has been done merely to increase execution speed for these very frequently used words. The routines that enter and exit from interrupts are also in assembly, in order to swap the background program in and out without disrupting foreground. In principle, a generic interrupt handler can be written and invoked through FORTH, at the price of slightly slower and more awkward performance. Instead, we patch the FORTH background driver directly into the entry and exit routines.

Another laboratory in the department uses a TRS- 80 Model I microcomputer with 32-KB memory, disk drives, and an LVB Corporation interface with input, output, and clock modules, running under MMS-FORTH. This system serves as a dedicated unit, running five simultaneous animal experiments on timing abilities, discrimination, and schedule performance. The realtime routines are similar to those described above, except that they employ a polling procedure, because 
vectored interrupts cannot easily be accessed on the TRS-80. The driver program operates in a continuous loop, servicing each experiment at least every $50 \mathrm{msec}$ ( $20 \mathrm{msec}$ is more typical). Niore experiments and faster servicing can be included. Very little effort has been made to optimize the component procedures. The only assembly code routines are those that mimic the TRS-80 BASIC "IN" and "OUT" commands.

\section{AVAILABILITY}

A list of FORTH vendors is reported by Williams (1980). More recent products are commonly advertised in trade publications.

The above MINC system is available from the author in exchange for two RX02-compatible diskettes, one for the MACRO source file and documentation and the other for the compiled language and a selection of FORTH programs. This system supports a VT105 terminal, a printer, and a modem on the standard serial line ports, and DEC digital input, digital output, and real-time clock modules. The documentation includes instructions for making minor modifications for a few different configurations. This version may be used either stand alone or under RT-11, although it cannot return to RT-11 after loading. An unmodified version of
PDP-11 FIG-FORTH, upon which this system is based, may be obtained from the FORTH Interest Group (FIG), Box 1105, San Carlos, California 94070. The group's most recent version differs from ours and meets the FORTH-79 standard. This language source is in the public domain. However, disk copies and documentation are sold (inexpensively) by private vendors. Contact FIG for details.

TRS-80 users may contact the author for a listing of the real-time programs running on MMS-FORTH (distributed by Miller Microcomputer Services, 61 Lake Shore Road, Natick, Massachusetts 01760). Other FORTH users are encouraged to contact the author in order to share programs and ideas.

\section{REFERENCES}

Katzan, H. Invitation to FORTH. New York: Petrocelli Books, 1981.

Ouverson, M. D. (Ed.). Dr. Dobb's journal for users of small computer systems. (Vol. 6, Issue 9). Menlo Park, Calif: People's Computer Company, 1981.

Williams, G. K. (Ed.). Byte (Vol. 5; Issue 8). New York: McGraw-Hill, 1980.

(Received for publication December 31, 1981; accepted January 5, 1982.) 